OPEN ACCESS

Edited by:

Xiaotian Wang,

Southwest University, China

Reviewed by:

Yuanzheng Chen,

Southwest Jiaotong University, China

Jihua Zhang,

Guizhou Provincial Key Laboratory of

Computational Nano-Material Science,

China

Daoxiong $W u$,

Hainan University, China

*Correspondence:

Zhaocai Zhang

zhangzhaocai@gmail.com

Xuefei Liu

201307129@gznu.edu.cn

Specialty section:

This article was submitted to Physical Chemistry and Chemical

Physics,

a section of the journal

Frontiers in Chemistry

Received: 27 July 2021 Accepted: 10 August 2021

Published: 01 October 2021

Citation:

Xiao W, Liu T, Zhang Y, Zhong Z, Zhang X, Luo Z, Lv B, Zhou X, Zhang Z and Liu X (2021) Tunable Schottky

Barrier and Interfacial Electronic

Properties in Graphene/

ZnSe Heterostructures.

Front. Chem. 9:744977.

doi: 10.3389/fchem.2021.744977

\section{Tunable Schottky Barrier and Interfacial Electronic Properties in Graphene/ZnSe Heterostructures}

\author{
Wenjun Xiao ${ }^{1}$, Tianyun Liu ${ }^{1}$, Yuefei Zhang ${ }^{1,2}$, Zhen Zhong ${ }^{1}$, Xinwei Zhang ${ }^{3}$, Zijiang Luo ${ }^{4}$, \\ Bing Lv ${ }^{1,2}$, Xun Zhou ${ }^{1,2}$, Zhaocai Zhang ${ }^{3 *}$ and Xuefei Liu ${ }^{1,2 *}$ \\ ${ }^{1}$ College of Physics and Electronic Science, Guizhou Normal University, Guiyang, China, ${ }^{2}$ Key Laboratory of Low Dimensional \\ Condensed Matter Physics of Higher Educational Institution of Guizhou Province, Guizhou Normal University, Guiyang, China, \\ ${ }^{3}$ Beijing Institute of Space Science and Technology Information, Beijing, China, ${ }^{4}$ College of Information, Guizhou University of \\ Finance and Economics, Guiyang, China
}

With a direct bandgap, two-dimensional (2D) ZnSe is a promising semiconductor material in photoelectric device fields. In this work, based on first-principles methods, we theoretically studied the modulation of the Schottky barrier height $(\mathrm{SBH})$ by applying horizontal and vertical strains on graphene/ZnSe heterojunction. The results show that the inherent electronic properties of graphene and ZnSe monolayers are both well-conserved because of the weak van der Waals ( $\mathrm{vdW}$ ) forces between two sublayers. Under horizontal strain condition, the n(p)-type SBH decreases from 0.56 (1.62) eV to $0.21(0.78) \mathrm{eV}$. By changing the interlayer distance in the range of $2.8 \AA$ to $4.4 \AA$, the $n(p)$-type $\mathrm{SBH}$ decreases (increases) from $0.88(0.98) \mathrm{eV}$ to $0.21(1.76) \mathrm{eV}$. These findings prove the $\mathrm{SBH}$ of the heterojunction to be tuned effectively, which is of great significance to optoelectronic devices, especially in graphene/ZnSe-based nano-electronic and optoelectronic devices.

Keywords: 2D heterojunction, Schottky barrier height, horizontal and vertical strain, bader charge, density function theory

\section{INTRODUCTION}

Ever since graphene was exfoliated experimentally in 2004 Novoselov et al. (2004), owing to its unique advantages, such as high charge mobility at room temperature and excellent Hall effect Novoselov et al. (2005), Zhang et al. (2005), it has attracted many theoretical and experimental attentions (Olabi et al., 2021; cao et al., 2018; Wang et al., 2020; Niu et al., 2020). Nevertheless, ZnSe as a zero bandgap material was not well-developed in graphene photonics and optoelectronics (Bonaccorso et al., 2010). In this regard, a lot of efforts have been devoted to exploring other novel two-dimensional (2D) crystal structures, and some new 2D materials were prepared (Naguib et al., 2013; Niu and Li, 2015; Liao et al., 2020). Among them, ZnSe is a direct bandgap 2D semiconductor material with a wide gap of $3.24 \mathrm{eV}$ by using Heyd-Scuseria-Ernzerhof (HSE) functional (Krukau et al., 2006), and it has attracted more and more attention in recent years. For instance, ZnSe was proved to be a potential material as inorganic scintillators Jagtap et al. (2019), as well as a cathode material of battery (Zhu et al., 2019). Besides, 2D ZnSe crystals have exhibited other interesting properties, including piezoelectric and dopant-induced semi-metallic tunable bandgap Liu et al. (2014), Yu et al. (2019), Khan et al. (2020), Sun et al. (2020), enabling its great potential applications in nano-electronics and optoelectronics fields. Furthermore, the energy bands of $\mathrm{ZnSe}$ meet the conditions of photocatalytic water splitting. Hence, it is also expected to be developed in new energy devices (Rubini et al., 2001; Hazrati et al., 2008; Yao et al., 2020). 
The van der Waals (vdW) heterostructure, as proposed by Geim and Grigorieva (2013), is composed of multiple 2D atomic layers without chemical bonds between them. Due to the weak interlayer vdW forces, heterojunction often retains the individual electronic and optical properties of a single layer, and some new physical properties may be obtained at the interface. Therefore, in recent years, the $2 \mathrm{D} \mathrm{vdW}$ heterojunction has attracted extensive attention in the field of electronic and optoelectronic devices (Zhang et al., 2020a; Guo et al., 2020; Zhu et al., 2021). Many scholars have studied vdW heterojunction based on $\mathrm{ZnSe}$, such as $\mathrm{AlP} / \mathrm{ZnSe}, \mathrm{ZnSe}_{2} / \mathrm{ZnSe}$, and CdS/ZnSe heterostructures (Dinger et al., 1999; Xiong and Zhou, 2019; Liu et al., 2020a). However, all the interfaces were constructed by two semiconductors, rather than metal/ semiconductor heterojunction. In fact, the interface properties of the semiconductor/metal heterojunction such as the charge transfer and Schottky barrier play a vital role in the device performance (Zhang et al., 2020b; Robertson et al., 2020; Chen et al., 2021). How is the charge transfer between a $2 \mathrm{D} \mathrm{ZnSe}$ nanosheet and graphene? How to tune the charge transfer and other electronic properties of the graphene/ZnSe interface? These questions have not been understood well yet. Hence, it is very essential to understand the interfacial properties of the ZnSe-based metal-semiconductor heterojunctions.

Besides, the 2D heterojunction Schottky barrier height ( $\mathrm{SBH}$ ) can be controlled through interlayer coupling, electric field, biaxial strain engineering, and atomic doping (Si et al., 2016; Zhang et al., 2018; Zhou et al., 2018; Zhang et al., 2020a; Nguyen et al., 2020; Liu et al., 2021). The modulation of SBH would improve the carrier transmission rate and change the type of Schottky contact. Although many studies on graphene-based vdW heterojunction have been published Georgiou et al. (2012), Si et al. (2016), Qiu et al. (2020), the electronic properties of the graphene/ZnSe heterostructure have not been clearly understood yet, as well as the SBH tunability under horizontal and vertical strain. In this study, we constructed a graphene/ZnSe heterojunction and calculated the electronic properties systematically. Our findings indicated the SBH of graphene/ZnSe could be effectively tuned by applying horizontal strain and vertical strain, which has potential applications in nano-mechanics, transistors, piezoelectric, and optoelectronics applications.

\section{COMPUTATIONAL METHODS}

In this study, all the calculations are based on the density functional theory (DFT) and projector augmented wave (PAW) Blöchl (1994), as carried out in the Vienna ab initio simulation package (VASP) (Kresse and Furthmüller, 1996). The Perdew-Burke-Ernzerhof (PBE) method based on the generalized gradient approximation (GGA) was used to describe exchange-correlation potential (Perdew et al., 1996). The geometric optimization and electronic property calculations were accomplished by applying an energy cutoff of $520 \mathrm{eV}$. The total energy convergence was set at $10^{-5} \mathrm{eV}$. The atomic structures considered were fully optimized until the convergence of force of $0.01 \mathrm{eV} / \AA$. The $\Gamma$-centered Monkhorst-Pack Monkhorst and Pack (1976) is used to sample the reciprocal space with a grid density of $5 \times 5 \times 1$. The weak interaction was corrected between sublayers by using Grimme's DFT-D3 method (Grimme, 2006). We used a vacuum thickness of $20 \AA$ to avoid bonding between the periodic sublayers (Liu et al., 2020b). Dipole correction was considered to reduce the error due to the asymmetry of the graphene/ZnSe interface along the $Z$-direction. The VASPKIT was used to do part of data post-processing (Wang et al., 2021).

The binding energy is one of the physical quantities that determine the heterojunction structural stability (Gélinas et al., 2011). Therefore, to verify the stability, we calculated the binding energies for graphene/ZnSe vdW heterostructure as follows (Zhao-Fu et al., 2014; Guo et al., 2020) (1):

$$
E_{b}=\frac{E_{\text {graphene/ZnSe }}-E_{\text {graphene }}-E_{\text {ZnSe }}}{A} .
$$

Here, $E_{b}$ is the heterojunction binding energy; $E_{\text {graphene/ZnSe }}$ represents the total energy of the heterostructure; $E_{\text {graphene }}$ and $E_{Z n S e}$ are the total energy of the graphene and $\mathrm{ZnSe}$ monolayer, separately; and $A$ is the interface area in the $\mathrm{x}-\mathrm{y}$ plane.

\section{RESULTS AND ANALYSIS}

\section{Structural Properties}

Before constructing the graphene/ $\mathrm{ZnSe}$ heterojunction, the lattice constants of graphene and $\mathrm{ZnSe}$ are relaxed to be $2.46 \AA$ and $4.07 \AA$, respectively, being in great agreement with the data in the literature (Priyadharsini et al., 2016; Yang et al., 2018). To reduce the mismatch, the graphene/ZnSe heterostructure was constructed by using a $5 \times 5$ supercell of graphene and a $3 \times$ 3 supercell of $\mathrm{ZnSe}$, as shown in Figure 1. It is noted that the lattice constants in the heterostructure for graphene and $\mathrm{ZnSe}$ are $12.3 \AA$ and $12.21 \AA$, leading to a lattice mismatch of only $0.73 \%$. To obtain the most stable heterojunction, we considered three stacking patterns based on the main high symmetry nature, as shown in Figure 2A. The binding energy of three stacking patterns is shown in Table 1 . The stack-III pattern with a binding energy of $-2.01 \mathrm{meVe}$ is the most stable heterojunction. Thus, in the following calculation, we only considered the stack-III pattern.

We further investigated the binding energy of stack-III pattern under different interlayer distances, as shown in Figure 2C. The results of relative energy indicated the most stable interlayer distance is $3.6 \AA$.

\section{Electrical Properties}

As expected, graphene shows a metallic nature with the Dirac point located at the $\mathrm{K}$ point and crossed by the Fermi energy level, as shown in Figure 3. As for $\mathrm{ZnSe}$, the valence band maximum (VBM) is at the $\Gamma$ point, as well as the conduction band minimum (CBM), indicating that $\mathrm{ZnSe}$ is a direct bandgap semiconductor, with a bandgap value of $2.11 \mathrm{eV}$ with PBE. According to the projected density of states (PDOS), the $\mathrm{VBM}$ of $\mathrm{ZnSe}$ was mainly contributed by the p-orbitals of $\mathrm{Zn}$ 


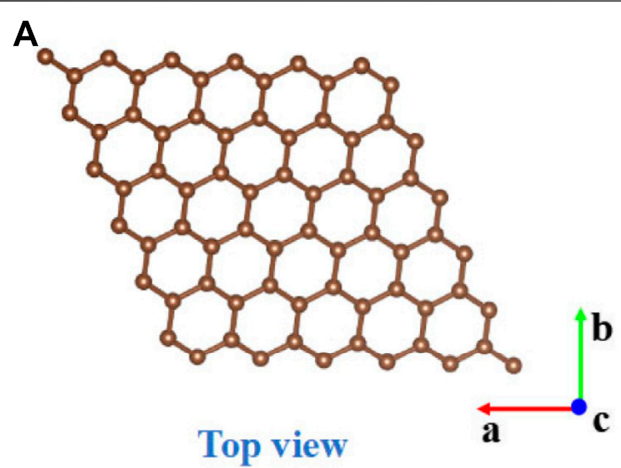

C $\odot$

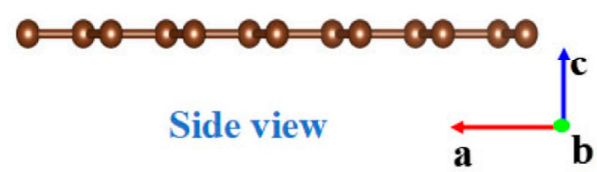

B<smiles>CC(C)O</smiles>

Se $\circ$ Zn $\odot$

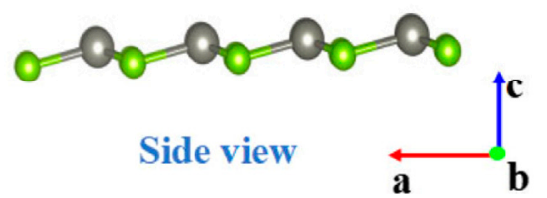

FIGURE 1 | (A) Top and side views of a $5 \times 5$ graphene, and (B) the top and side views of a $3 \times 3$ ZnSe supercell. The brown spheres represent C, the gray spheres represent $\mathrm{Zn}$, and the green spheres represent Se.

A

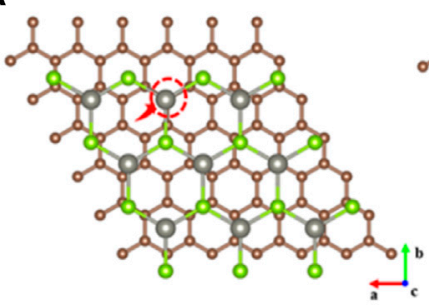

Stack-I

B

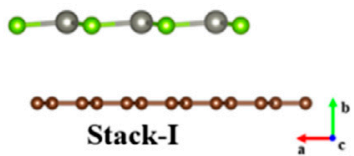

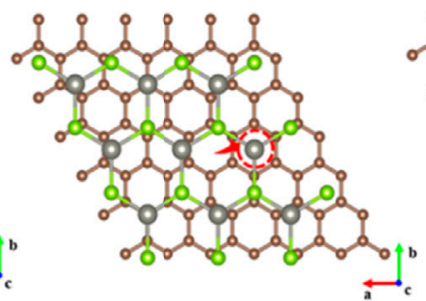

Stack-II

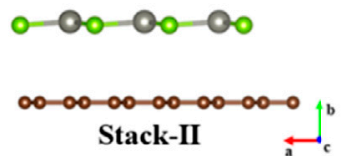

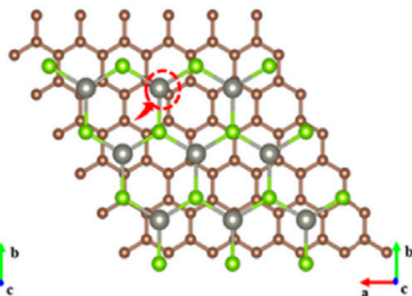

Stack-III
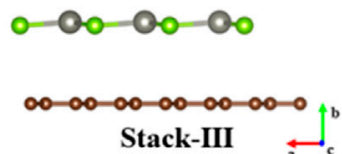

C

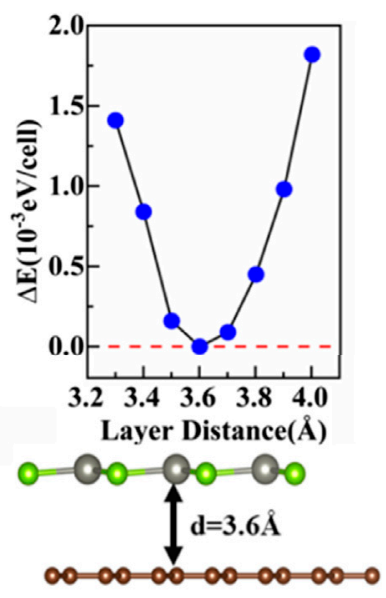

FIGURE 2 | (A), (B) Three stacked patterns of top and side views. (C) Relationship between layer spacing and binding energy of graphene/ZnSe.

TABLE 1 | Binding energy of three stacking patterns.

\begin{tabular}{lcccc}
\hline Stack type & $\mathbf{E}_{\text {graphene/ }} / \mathbf{Z n S e}(\mathbf{e V})$ & $\boldsymbol{E}_{\text {graphene }}(\mathbf{e V})$ & $\boldsymbol{E}_{\mathbf{Z n S e}}(\mathbf{e V})$ & -49.41 \\
\hline Stack-I & -514.61 & -461.65 & -49.41 \\
Stack-II & -514.70 & -461.65 & -1.77 \\
Stack-III & -515.11 & -461.65 & -49.41
\end{tabular}

and Se, while the CBM was mainly contributed by the s-orbital electrons of $\mathrm{Zn}$.

As well known, the PBE method would underestimate the bandgap of semiconductors, and the HSE method can solve this problem extremely well. However, both the PBE and HSE would predict the same band structure shape and variation trends of the ZnSe monolayer under different strains, with limited resources, so we use the PBE method to calculate all properties of the 

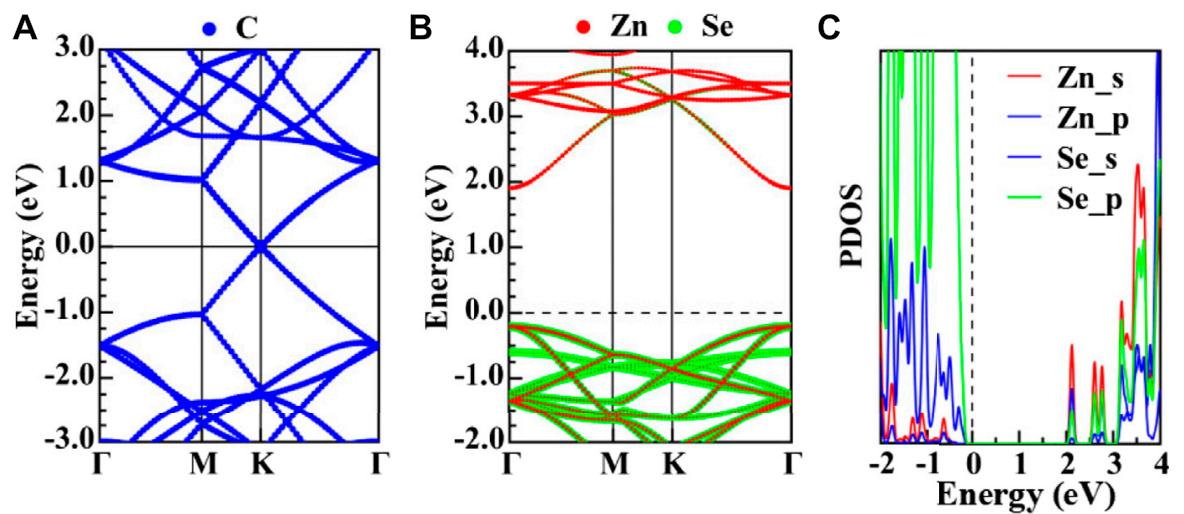

FIGURE 3 | (A) Energy band diagram of graphene. (B), (C) Projected energy band diagram and density of states of ZnSe, respectively.

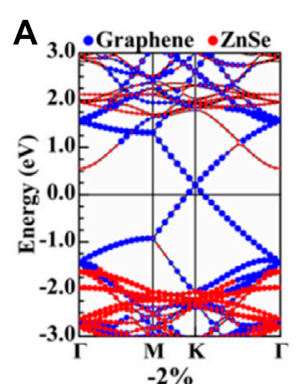

$\mathbf{F}$

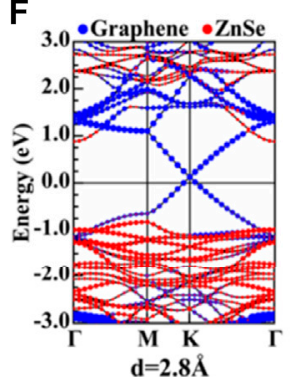

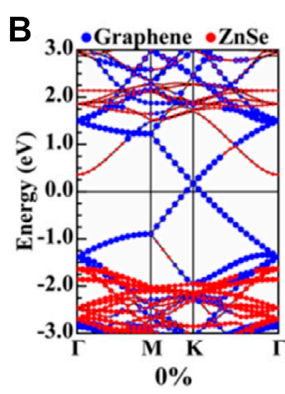

G

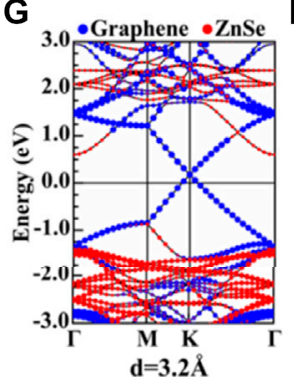

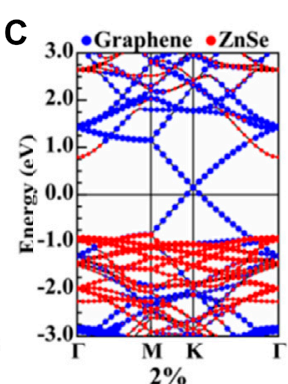

$\mathrm{H}$

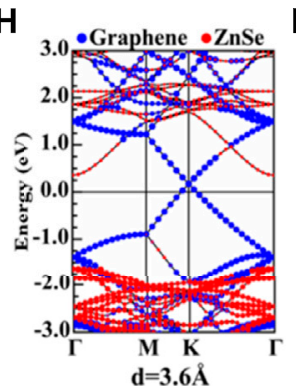

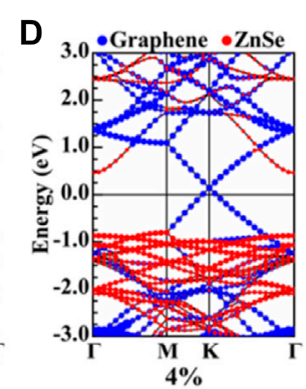

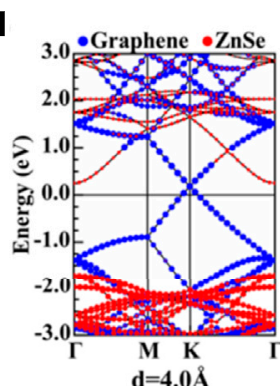

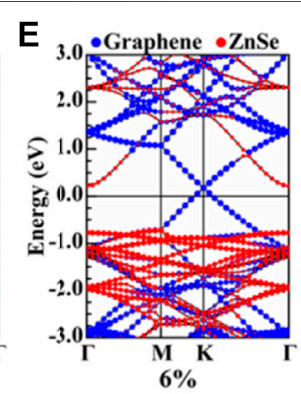

J

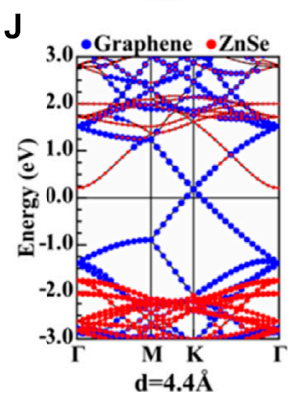

FIGURE 4 | (A-E) Projected band structures of the graphene/ZnSe heterojunction under horizontal strain. (F-J) Projected band structures of the graphene/ZnSe heterojunction under vertical strain.

considered systems. Next, we further researched the electronic properties of the graphene/ZnSe heterojunction by figuring out the band structures and density of states under different horizontal or vertical strains (Phuc et al., 2017).

In this study, we applied horizontal strain from $-2 \%$ to $+6 \%$ on the graphene/ZnSe heterojunction, with a span of $2 \%$. The positive value indicates tensile strain, while the negative value represents compressive strain. The reason why more negative strains are not considered is that a much larger strain is hard to be experimentally achieved, and it will also make the nanomaterials nonstable (Liao et al., 2020). When the compressive strain is less than $-2 \%$, the heterojunction optimization was failed. In general, tensile strain is relatively easier than that of compressive strain to implement in engineering. Hence, we only consider the $-2 \%$ horizontal compression strain but a tensile strain of $6 \%$. The projected band structures of the graphene/ZnSe heterojunction under different horizontal strains are shown in Figures 4A-E. From the figure, the Dirac point is well-maintained and the Fermi level is fixed at the $\mathrm{K}$ point. With the increase in the tensile strain from 0 to $6 \%$, the bandgap decreases from 1.98 to $0.99 \mathrm{eV}$, and the bandgap always maintains a direct bandgap. The electron transfer ability of graphene to $\mathrm{ZnSe}$ is weakened, with the increased horizontal tensile strain smaller than $4 \%$. When the horizontal tensile strain is larger than $4 \%$, the electron transfer ability of graphene to $\mathrm{ZnSe}$ is enhanced. When horizontal or vertical compressive strain is applied, the CBM of $\mathrm{ZnSe}$ is moved up. Additionally, it seems that the horizontal compressive strain has a smaller effect than vertical strain on the VBMs, as shown in 

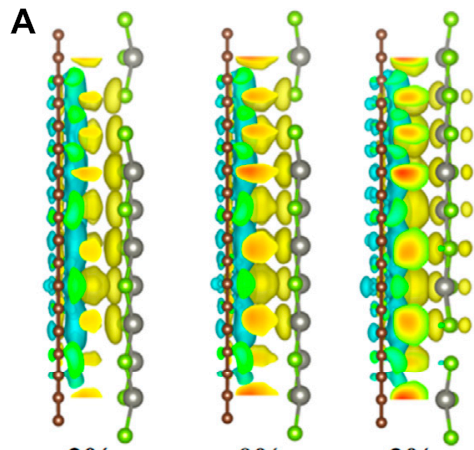

$-2 \%$

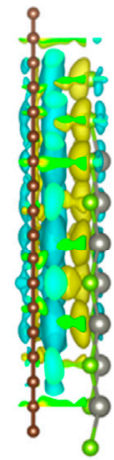

$\mathrm{d}=\mathbf{2 . 8 \AA}$
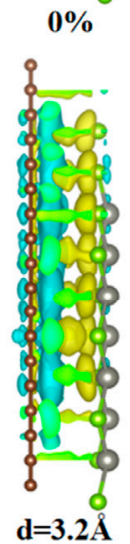

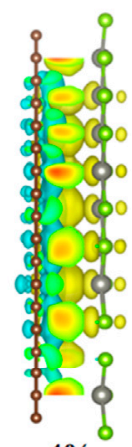

$4 \%$
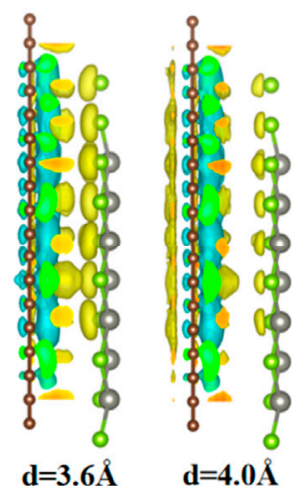
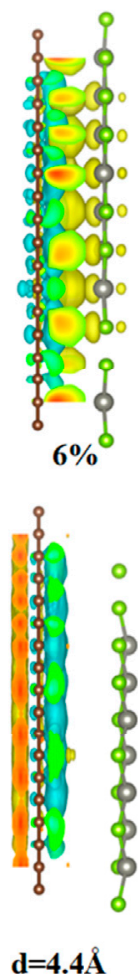
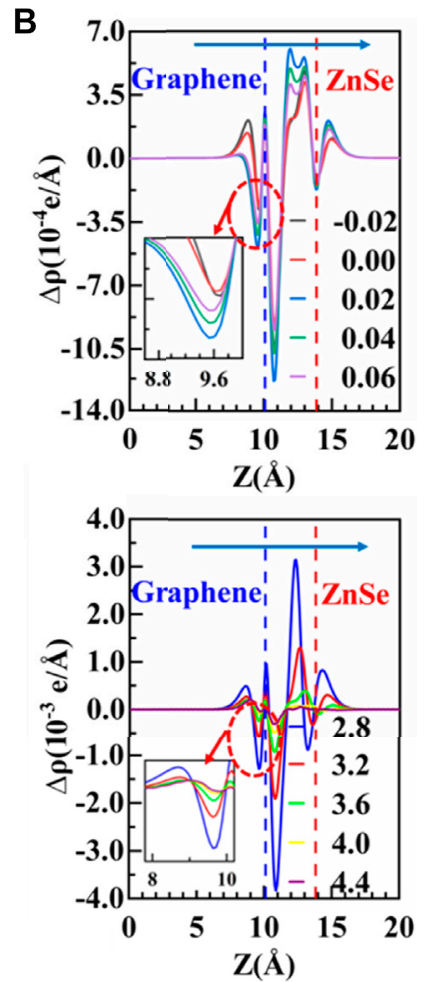

FIGURE 5 | (A) On behalf of the charge density difference of the graphene/ZnSe system under different horizontal strains and vertical strain, yellow and cyan represent the accumulation and depletion of electrons, respectively. (B) PCDD under horizontal strain and vertical strain.

Figures 4A, F, G. Under tensile strain, both the VBMs and CBMs would shift up (down) for horizontal (vertical) cases, except for a horizontal strain of $6 \%$ (see Figure 4E). These different changes of CBM and VBM would lead to the bandgap change with the external strains. The results mean that both the vertical and horizontal strain plays a pivotal role in tuning the electronic properties of the graphene/ZnSe heterojunction. These phenomenons are resulting from the charge redistribution between the two sublayers under different strains (Liu et al., 2019; Liu et al., 2020b), as verified in Figure 5.

Next, to investigate the charge transfers and charge redistribution more clearly, the charge density difference of the graphene/ZnSe heterojunction was analyzed under different horizontal and vertical strains, as shown in Figure 5A. The charge density difference shows charge accumulation in the interface region near $\mathrm{ZnSe}$ and depletion in the interface region close to the graphene, which suggests a charge transfer from graphene to $\mathrm{ZnSe}$. But, the variations in charge redistributions under different strain conditions are found, which leads to the different change regularity of band structures.

To see the charge redistribution, the plane-averaged charge density difference (PCDD) was calculated as follows (Zhang et al., 2015):

$$
\Delta \rho=\rho_{\text {graphenelZnSe }}-\rho_{\text {graphene }}-\rho_{\text {ZnSe }}
$$

In this case, $\rho_{\text {graphenelZnse }}, \rho_{\text {graphene }}$, and $\rho_{\text {Znse }}$ are the PCDD of the graphene/ZnSe heterojunction, the isolated graphene single layer, and the isolated $\mathrm{ZnSe}$, respectively. The PCDD curve is depicted in Figure 5B; when the horizontal strain is applied, charge transfer is tunable and agrees with the results of Bader charge analysis (Henkelman et al., 2006), as shown in Figure 6A, the charge is mainly concentrated on the surface near the $\mathrm{ZnSe}$ of layers. While the vertical strain is applied, as expected, the smaller the interlayer distance, the stronger coupling between $\mathrm{ZnSe}$ and graphene was found, leading to more electrons being transferred from graphene to $\mathrm{ZnSe}$. The PCDD curve also proves the accuracy of the analysis of the band structure results.

In Figure 7C, we further depict the plane-averaged electrostatic potentials of the graphene/ZnSe interface under different horizontal and vertical strains. The graphene layer has a deeper potential than the ZnSe layer. The potential difference of the graphene/ZnSe heterostructure is $11.92 \mathrm{eV}$ under an equilibrium distance of $\mathrm{d}=3.6 \AA$, which further proves that the electrons are transferred from graphene to $\mathrm{ZnSe}$. As shown in Figure 7A, horizontal strain ranges from $-2 \%$ to $+6 \%$, the potential difference between graphene and $\mathrm{ZnSe}$ monolayers is tuned effectively, indicating that the ability of charge transfer can be modulated by applying horizontal strain. At the tensile strain of $2 \%$, the potential difference reached $12.26 \mathrm{eV}$, indicating that the graphene/ZnSe vdW heterojunction should be able to maintain the high carrier mobility of graphene and promote the development of new high-performance nano-electronic devices. As shown in Figure $\mathbf{7 B}$, under a vertical strain, it shows the potential 

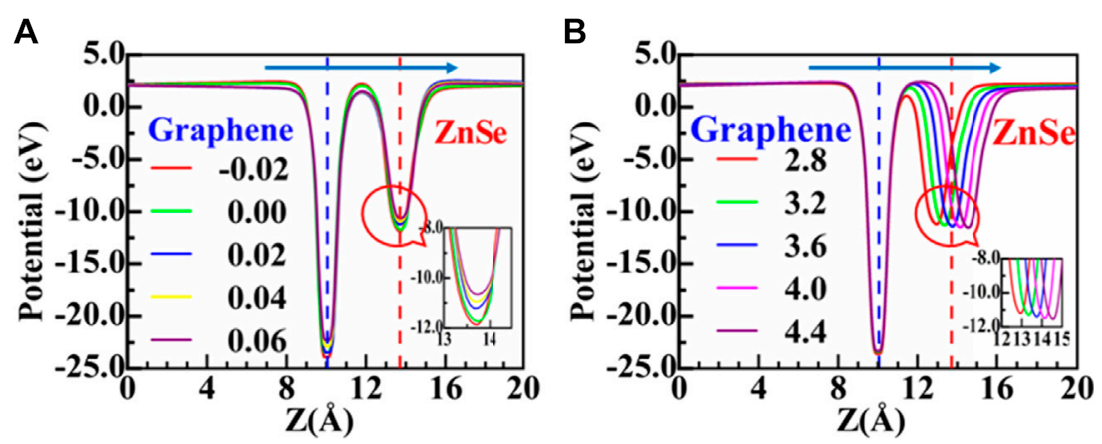

FIGURE 6 | (A, D) Variation trend of CBM, VBM, and Fermi energy levels $E_{F}$ with applied strain. (B, E) Variation of $\Phi_{n}$, $\Phi_{p}$, bandgap with the strain. (C, F) Amount of electron transfer from graphene to $\mathrm{ZnSe}$ as a function of the strain.
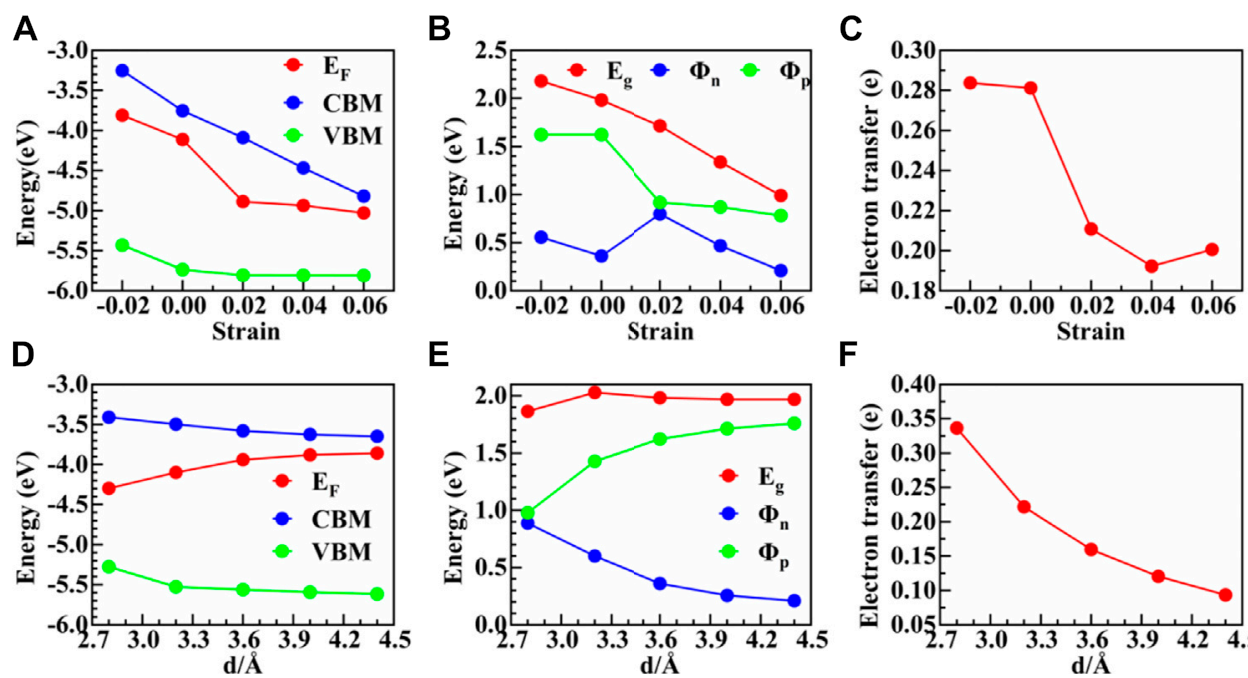

E
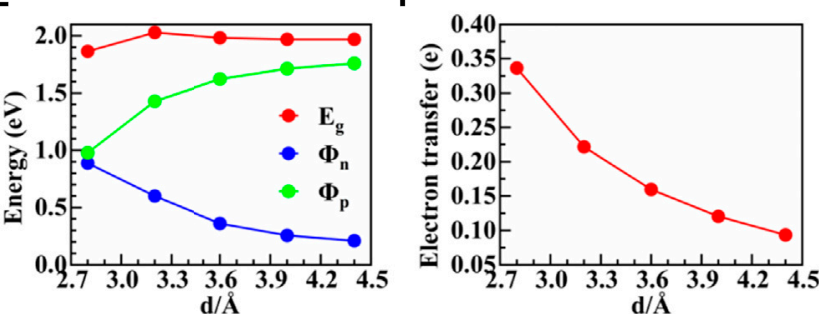

FIGURE 7 | (A), (B) Average electrostatic potential along the $z$-direction for the heterostructure under different horizontal strains and vertical strains, respectively.

difference between graphene and $\mathrm{ZnSe}$ under an interlayer distance of $2.8 \AA$ and $4.4 \AA$ is 12.38 and $11.78 \mathrm{eV}$, respectively. In other words, with the increase in the interlayer distance, the potential differences between graphene and ZnSe decrease, leading to the charge transfer from the graphene layer to the ZnSe layer being reduced, which is confirmed by the Bader charge analysis shown in Figure 6B.

\section{Tunability of SBH Under Strain}

The SBH of the metal/semiconductor interfacial system is an important parameter (Zhang et al., 2020b; Robertson et al., 2020; Chen et al., 2021). Therefore, it is necessary to study the SBH of the graphene/ZnSe interface to better understand this heterojunction.

Based on Bardeeen's (Bardeen, 1947) Schottky-Mott model of metal/semiconductor heterostructures, the n-type and p-type Schottky barriers are determined as follows:

$$
\Phi_{n}=\mathrm{CBM}-\mathrm{E}_{F}
$$

$$
\Phi_{p}=\mathrm{E}_{F}-\mathrm{VBM}
$$

where the $\Phi_{n}$ denotes n-type $\mathrm{SBH}, \Phi_{p}$ is p-type $\mathrm{SBH}$, and $\mathrm{E}_{\mathrm{F}}$ is the Fermi level. Without any horizontal or vertical strain, $\Phi_{n}$ and $\Phi_{p}$ of the graphene/ZnSe heterostructure are 0.36 and $1.62 \mathrm{eV}$, respectively, indicating that graphene/ZnSe vdW $\left(\Phi_{n}<\Phi_{p}\right)$. As is well known, $\mathrm{SBH}$ and contact types of the heterojunction can be controlled by applying strain (Liu et al., 2019; Liu et al., 2020c). Therefore, we further investigate the effects of SBH and its tunability of the graphene/ZnSe heterojunction by applying the horizontal and vertical strains.

As shown in Figure 7A, the results indicate that CBM and VBM are decreased (increased) with the increase in horizontal tensile (compressive) strain and the Fermi energy level, but with a different change degree. Thus, the heterojunction bandgap was adjusted in a large range, indicating that the horizontal strain is an effective method of regulating the $\mathrm{SBH}$. As is depicted more intuitively in Figure $7 \mathbf{B}$, the results show both the n-type and p-type SBH was changed obviously under different horizontal strains. For instance, 
the n-type $\mathrm{SBH}$ can change from 0.56 to $0.21 \mathrm{eV}$, and the p-type SBH is decreased from 1.62 to $0.78 \mathrm{eV}$ according to the applied strain values, but the $\mathrm{n}$-type $\mathrm{SBH}$ contact is always maintained. Although the Ohmic contact is not realized, the large decrease in SBH would enhance the performance of the graphene/ZnSe-based nanodevices.

It should be noted that variation of heterojunction layer spacing can be achieved by experimental techniques (Zhang et al., 2014), which have been diffusely used to regulate the electronic properties and SBH of the vdW heterostructures. In this study, to understand how vertical strain regulates the graphene/ZnSe heterojunction $\mathrm{SBH}$, the interlayer distance of the graphene/ZnSe heterojunction was changed from $2.8 \AA$ to $4.4 \AA$. In Figures 7D, E, the VBM and CBM are decreased with the increase in the interlayer distance. In contrast, the Fermi energy level is shifted up until the interlayer is larger than 3.6 $\AA$, leading to the SBH of n-type and p-type changing obviously, as shown in Figure 7E, and by changing the interlayer distance in the range of $2.8 \AA$ to $4.4 \AA$, the n(p)-type SBH decreases (increases) from $0.88(0.98) \mathrm{eV}$ to $0.21(1.76) \mathrm{eV}$. Thus, for the graphene/ZnSe heterostructure, the n-type Schottky is still maintained. Based on our results in Figure 7E, it can be informed that an n-type-to-p-type contact would be formed if the interlayer distance is smaller enough. Therefore, after the strain adjustment, graphene can be used as an ideal electrode material for $\mathrm{ZnSe}$, and the SBH can be significantly tuned by applying both lateral and vertical strains, which is expected in the graphene/ZnSe-based Schottky devices.

\section{CONCLUSION}

In conclusion, we have systematically studied the electronic properties and the efficient modulations of SBH of the $\mathrm{vdW}$ graphene/ZnSe heterostructure by DFT calculations. The band

\section{REFERENCES}

Bardeen, J. (1947). Surface States and Rectification at a Metal Semi-conductor Contact. Phys. Rev. 71, 82-105. doi:10.1103/physrev.71.717

Blöchl, P. E. (1994). Projector Augmented-Wave Method. Phys. Rev. B. 50, 17953-17979. doi:10.1103/physrevb.50.17953

Bonaccorso, F., Sun, Z., Hasan, T., and Ferrari, A. C. (2010). Graphene Photonics and Optoelectronics. Nat. Photon. 4, 611-622. doi:10.1038/nphoton.2010.186

cao, Y., Fatemi, V., Fang, S., Watanabe, K., Taniguchi, T., Kaxiras, E., et al. (2018). Unconventional Superconductivity in Magic-Angle Graphene Superlattices. Nature 556, 43-50. doi:10.1038/nature26160

Chen, J., Zhang, Z., Guo, Y., and Robertson, J. (2021). Schottky Barrier Heights of Defect-free metal/ZnO, CdO, MgO, and SrO Interfaces. J. Appl. Phys. 129, 175304. doi:10.1063/5.0047447

Dinger, A., Petillon, S., Grün, M., Hetterich, M., and Klingshirn, C. (1999). Conduction Band Offset of the CdS/ZnSe Heterostructure. Semicond. Sci. Technol. 14, 595-598. doi:10.1088/0268-1242/14/7/301

Geim, A. K., and Grigorieva, I. V. (2013). Van der Waals heterostructures. Nature 499, 419-425. doi:10.1038/nature12385

Gélinas, S., Paré-Labrosse, O., Brosseau, C.-N., Albert-Seifried, S., McNeill, C. R., Kirov, K. R., et al. (2011). The Binding Energy of Charge-Transfer Excitons Localized at Polymeric Semiconductor Heterojunctions. J. Phys. Chem. C. 115, 7114-7119. doi:10.1021/jp200466y

Georgiou, T., Jalil, R., Belle, B. D., Britnell, L., Gorbachev, R. V., Morozov, S. V., et al. (2012). Vertical Field-Effect Transistor Based on Graphene-WS2 Heterostructures for Flexible and Transparent Electronics. Nat. Nanotech. 8, 100-103. doi:10.1038/nnano.2012.224 structures, the charge density differences, and the Bader charge transfer are studied in detail. The results show that the electrons will be transformed from graphene to $\mathrm{ZnSe}$, and the transfer amount can be tuned effectively by applying both horizontal and vertical strains. As a consequence, the positions of CBM and VBM as well as Fermi energy level will be changed with the strain, and thus, the SBH is modulated effectively. These findings would provide useful guidance for designing controllable graphene/ZnSe-based Schottky nanodevices.

\section{DATA AVAILABILITY STATEMENT}

The original contributions presented in the study are included in the article/supplementary material; further inquiries can be directed to the corresponding authors.

\section{AUTHOR CONTRIBUTIONS}

WX: investigation, conceptualization, methodology, data curation, visualization, validation, and writing; TL: project administration and writing-reviewing and editing; YZ: visualization; ZZ: editing; XWZ: validation; ZL: visualization and project administration; BL: software; XZ: data curation; ZCZ: writing-reviewing and editing; and XL: project administration and writing-reviewing and editing.

\section{FUNDING}

This work is supported by the National Natural Science Foundation of China (Grant No. 12164009).

Grimme, S. (2006). Semiempirical GGA-type Density Functional Constructed with a Long-Range Dispersion Correction. J. Comput. Chem. 27, 1787-1799. doi:10.1002/jcc.20495

Guo, H., Zhang, Z., Huang, B., Wang, X., Niu, H., Guo, Y., et al. (2020). Theoretical study on the photocatalytic properties of $2 \mathrm{D} \operatorname{InX}(\mathrm{X}=\mathrm{S}$, Se)/transition metal disulfide (MoS2 and WS2) van der Waals heterostructures. Nanoscale 12, 20025-20032. doi:10.1039/d0nr04725b

Hazrati, E., Hashemifar, S. J., and Akbarzadeh, H. (2008). First Principles Study of Bulk CrSe and CrSe/ZnSe(001) Interface. J. Appl. Phys. 104, 113719. doi:10.1063/1.3039510

Henkelman, G., Arnaldsson, A., and Jónsson, H. (2006). A Fast and Robust Algorithm for Bader Decomposition of Charge Density. Comput. Mater. Sci. 36, 354-360. doi:10.1016/j.commatsci.2005.04.010

Jagtap, S., Chopade, P., Tadepalli, S., Bhalerao, A., and Gosavi, S. (2019). A Review on the Progress of ZnSe as Inorganic Scintillator. Opto-Electronics Rev. 27, 90-103. doi:10.1016/j.opelre.2019.01.001

Khan, M. S., Shi, L., and Zou, B. (2020). Impact of Vacancy Defects on Optoelectronic and Magnetic Properties of Mn-Doped ZnSe. Comput. Mater. Sci. 174, 109493. doi:10.1016/j.commatsci.2019.109493

Kresse, G., and Furthmüller, J. (1996). Efficient Iterative Schemes Forab InitiototalEnergy Calculations Using a Plane-Wave Basis Set. Phys. Rev. B. 54, 11169-11186. doi:10.1103/physrevb.54.11169

Krukau, A. V., Vydrov, O. A., Izmaylov, A. F., and Scuseria, G. E. (2006). Influence of the Exchange Screening Parameter on the Performance of Screened Hybrid Functionals. J. Chem. Phys. 125, 224106. doi:10.1063/1.2404663

Liao, Y., Zhang, Z., Gao, Z., Qian, Q., and Hua, M. (2020). Tunable Properties of Novel Ga2O3 Monolayer for Electronic and Optoelectronic Applications. ACS Appl. Mater. Inter. 12, 30659-30669. doi:10.1021/ acsami.0c04173 
Liu, C., Zhang, W., Sun, J., Wen, J., Yang, Q., Cuo, H., et al. (2014). Piezoelectric Nanogenerator Based on a Flexible Carbon-fiber/ZnO-ZnSe Bilayer Structure Wire. Appl. Surf. Sci. 322, 95-100. doi:10.1016/j.apsusc.2014.10.081

Liu, P., Han, J., Zhu, K., Dong, Z., and Jiao, L. (2020). Heterostructure SnSe 2/ ZnSe@PDA Nanobox for Stable and Highly Efficient Sodium-Ion Storage. Adv. Energ. Mater. 10, 2000741. doi:10.1002/aenm.202000741

Liu, X., Gao, Z., Wang, V., Luo, Z., Lv, B., Ding, Z., et al. (2020). Extrapolated Defect Transition Level in Two-Dimensional Materials: The Case of Charged Native Point Defects in Monolayer Hexagonal Boron Nitride. ACS Appl. Mater. Inter. 12, 17055-17061. doi:10.1021/acsami.9b23431

Liu, X., Lv, B., Ding, Z., and Luo, Z. (2020). Van der Waals heterostructure of graphene and As2S3: Tuning the Schottky barrier height by vertical strain. J. Cryst. Growth. 549, 125882. doi:10.1016/j.jcrysgro.2020.125882

Liu, X., Zhang, Z., Luo, Z., Lv, B., and Ding, Z. (2019). Tunable Electronic Properties of Graphene/g-AlN Heterostructure: The Effect of Vacancy and Strain Engineering. Nanomaterials 9, 1674-1688. doi:10.3390/nano9121674

Liu, X., Zhang, Z., Lv, B., Ding, Z., and Luo, Z. (2021). Impact of the Vertical Strain on the Schottky Barrier Height for graphene/AIN Heterojunction: a Study by the FirstPrinciples Method. Eur. Phys. J. B. 94, 1-7. doi:10.1140/epjb/s10051-020-00010-w

Monkhorst, H. J., and Pack, J. D. (1976). Special Points for Brillouin-Zone Integrations. Phys. Rev. B. 13, 5188-5192. doi:10.1103/physrevb.13.5188

Naguib, M., Mochalin, V. N., Barsoum, M. W., and Gogotsi, Y. (2013). 25th Anniversary Article: MXenes: A New Family of Two-Dimensional Materials. Adv. Mater. 26, 992-1005. doi:10.1002/adma.201304138

Nguyen, C. V., Idrees, M., Phuc, H. V., Hieu, N. N., Binh, N. T. T., Amin, B., et al. (2020). Interlayer Coupling and Electric Field Controllable Schottky Barriers and Contact Types in Graphene/PbI2 Heterostructures. Phys. Rev. B. 101, 235419. doi:10.1103/physrevb.101.235419

Niu, H., Zhang, Z., Wang, X., Wan, X., Shao, C., and Guo, Y. (2020). Theoretical Insights into the Mechanism of Selective Nitrate-to-Ammonia Electroreduction on SingleAtom Catalysts. Adv. Funct. Mater. 31, 2008533. doi:10.1002/adfm.202008533

Niu, T., and Li, A. (2015). From Two-Dimensional Materials to Heterostructures. Prog. Surf. Sci. 90, 21-45. doi:10.1016/j.progsurf.2014.11.001

Novoselov, K. S., Geim, A. K., Morozov, S. V., Jiang, D., Katsnelson, M. I., Grigorieva, I. V., et al. (2005). Two-dimensional Gas of Massless Dirac Fermions in Graphene. Nature 438, 197-200. doi:10.1038/nature04233

Novoselov, K. S., Geim, A. K., Morozov, S. V., Jiang, D., Zhang, Y., Dubonos, S. V., et al. (2004). Electric Field Effect in Atomically Thin Carbon Films. Science 306, 666-669. doi:10.1126/science.1102896

Olabi, A. G., Abdelkareem, M. A., Wilberforce, T., and Sayed, E. T. (2021). Application of Graphene in Energy Storage Device - A Review. Renew. Sust. Energ. Rev. 135, 110026. doi:10.1016/j.rser.2020.110026

Perdew, J. P., Burke, K., and Ernzerhof, M. (1996). Generalized Gradient Approximation Made Simple. Phys. Rev. Lett. 77, 3865-3868. doi:10.1103/physrevlett.77.3865

Phuc, H. V., Hieu, N. N., Hoi, B. D., Phuong, L. T. T., Hieu, N. V., and Nguyen, C. V. (2017). Out-of-plane Strain and Electric Field Tunable Electronic Properties and Schottky Contact of Graphene/antimonene Heterostructure. Superlattices. Microstructures 112, 554-560. doi:10.1016/j.spmi.2017.10.011

Priyadharsini, N., Elango, M., Vairam, S., Venkatachalam, T., and Thamilselvan, M. (2016). Effect of Temperature and $\mathrm{pH}$ on Structural, Optical and Electrical Properties of Ni Doped ZnSe Nanoparticles. Optik 127, 7543-7549. doi:10.1016/j.ijleo.2016.05.079

Qiu, B., Zhao, X. W., Hu, G. C., Yue, W. W., Yuan, X. B., and Ren, J. F. (2020). Tuning Optical Properties of Graphene/WSe2 Heterostructure by Introducing Vacancy: First Principles Calculations. Physica E: Low-dimensional Syst. Nanostructures. 116, 113729. doi:10.1016/j.physe.2019.113729

Robertson, J., Guo, Y., Zhang, Z., and Li, H. (2020). Extending the Metal-Induced gap State Model of Schottky Barriers. J. Vacuum Sci. Tech. B. 38, 042208. doi:10.1116/6.0000164

Rubini, S., Milocco, E., Sorba, L., Pelucchi, E., Franciosi, A., Garulli, A., et al. (2001). Structural and Electronic Properties of ZnSe/AlAs Heterostructures. Phys. Rev. B. 63, 155312. doi:10.1103/physrevb.63.155312

Si, C., Lin, Z., Zhou, J., and Sun, Z. (2016). Controllable Schottky Barrier in GaSe/ graphene Heterostructure: the Role of Interface Dipole. 2d Mater. 4, 015027. doi:10.1088/2053-1583/4/1/015027

Sun, C., Chen, X., Hong, R., Li, X., Xu, X., Chen, X., et al. (2020). Enhancing the Photoelectrical Performance of graphene/4H-SiC/graphene Detector by Tuning a Schottky Barrier by Bias. Appl. Phys. Lett. 117, 071102. doi:10.1063/5.0012566
Wang, V., Xu, N., Liu, J.-C., Tang, G., and Geng, W.-T. (2021). VASPKIT: A UserFriendly Interface Facilitating High-Throughput Computing and Analysis Using VASP Code. Comp. Phys. Commun. 267, 108033. doi:10.1016/j.cpc.2021.108033

Wang, X., Niu, H., Liu, Y., Shao, C., Robertson, J., Zhang, Z., et al. (2020). Theoretical Investigation on Graphene-Supported Single-Atom Catalysts for Electrochemical CO2 Reduction. Catal. Sci. Technol. 10, 8465-8472. doi:10.1039/d0cy01870h

Xiong, A., and Zhou, X. (2019). Tunable electronic and optical properties of novel ZnSe/AlP van der Waals heterostructure. Mater. Res. Express. 6, 075907. doi:10.1088/2053-1591/ab15ea

Yang, H., Li, J., Yu, L., Huang, B., Ma, Y., and Dai, Y. (2018). A Theoretical Study on the Electronic Properties of In-Plane CdS/ZnSe Heterostructures: Type-II Band Alignment for Water Splitting. J. Mater. Chem. A. 6, 4161-4166. doi:10.1039/c7ta10624f

Yao, F., Zhou, X., and Xiong, A. (2020). Tunable electronic and optical properties of two-dimensional ZnSe/AlAs van der Waals heterostructure. Appl. Phys. A. 126, 1-10. doi:10.1007/s00339-020-03674-4

Yu, J. H., Kim, J., Hyeon, T., and Yang, J. (2019). Facile Synthesis of Manganese (II)-doped ZnSe Nanocrystals with Controlled Dimensionality. J. Chem. Phys. 151, 244701. doi:10.1063/1.5128511

Zhang, X., Meng, F., Christianson, J. R., Arroyo-Torres, C., Lukowski, M. A., Liang, D., et al. (2014). Vertical Heterostructures of Layered Metal Chalcogenides by van der Waals Epitaxy, Nano Lett. 14, 3047-3054. doi:10.1021/nl501000k

Zhang, Y., Tan, Y.-W., Stormer, H. L., and Kim, P. (2005). Experimental Observation of the Quantum Hall Effect and Berry's Phase in Graphene. Nature 438, 201-204. doi:10.1038/nature04235

Zhang, Z., Cao, R., Wang, C., Li, H.-B., Dong, H., Wang, W.-h., et al. (2015). GaN as an Interfacial Passivation Layer: Tuning Band Offset and Removing Fermi Level Pinning for III-V MOS Devices. ACS Appl. Mater. Inter. 7, 5141-5149. doi:10.1021/am507287f

Zhang, Z., Guo, Y., and Robertson, J. (2020). Origin of Weaker Fermi Level Pinning and Localized Interface States at Metal Silicide Schottky Barriers. J. Phys. Chem. C 124, 19698-19703. doi:10.1021/acs.jpcc.0c06228

Zhang, Z., Huang, B., Qian, Q., Gao, Z., Tang, X., and Li, B. (2020). Strain-tunable III-nitride/ZnO Heterostructures for Photocatalytic Water-Splitting: A Hybrid Functional Calculation. APL Mater. 8, 041114. doi:10.1063/5.0005648

Zhang, Z., Qian, Q., Li, B., and Chen, K. J. (2018). Interface Engineering of Monolayer MoS2/GaN Hybrid Heterostructure: Modified Band Alignment for Photocatalytic Water Splitting Application by Nitridation Treatment. ACS Appl. Mater. Inter. 10, 17419-17426. doi:10.1021/acsami.8b01286

Zhao-Fu, Z., Tie-Ge, Z., Hai-Yang, Z., and Xiang-Lei, W. (2014). First-principles Calculations of 5d Atoms Doped Hexagonal-AlN Sheets: Geometry, Magnetic Property and the Influence of Symmetry and Symmetry-Breaking on the Electronic Structure. Chin. Phys. B. 23, 016801. doi:10.1088/1674-1056/23/1/016801

Zhou, W., Guo, Y., Liu, J., Wang, F. Q., Li, X., and Wang, Q. (2018). 2D SnSe-Based vdW Heterojunctions: Tuning the Schottky Barrier by Reducing Fermi Level Pinning. Nanoscale 10, 13767-13772. doi:10.1039/c8nr02843e

Zhu, L., Wang, Z., Wang, L., Xie, L., Li, J., and Cao, X. (2019). ZnSe Embedded in N-Doped Carbon Nanocubes as Anode Materials for High-Performance Li-Ion Batteries. Chem. Eng. J. 364, 503-513. doi:10.1016/j.cej.2019.01.191

Zhu, X. T., Xu, Y., Cao, Y., Zhao, Y. Q., Sheng, W., Nie, G.-Z., et al. (2021). Investigation of the Electronic Structure of Two-Dimensional GaN/Zr2CO2 hetero-junction: Type-II Band Alignment with Tunable Bandgap. Appl. Surf. Sci. 542, 148505. doi:10.1016/j.apsusc.2020.148505

Conflict of Interest: The authors declare that the research was conducted in the absence of any commercial or financial relationships that could be construed as a potential conflict of interest.

Publisher's Note: All claims expressed in this article are solely those of the authors and do not necessarily represent those of their affiliated organizations, or those of the publisher, the editors, and the reviewers. Any product that may be evaluated in this article, or claim that may be made by its manufacturer, is not guaranteed or endorsed by the publisher.

Copyright (c) 2021 Xiao, Liu, Zhang, Zhong, Zhang, Luo, Lv, Zhou, Zhang and Liu. This is an open-access article distributed under the terms of the Creative Commons Attribution License (CC BY). The use, distribution or reproduction in other forums is permitted, provided the original author(s) and the copyright owner(s) are credited and that the original publication in this journal is cited, in accordance with accepted academic practice. No use, distribution or reproduction is permitted which does not comply with these terms. 